\title{
IMPACTS OF CONNECTED AND AUTOMATED VEHICLES ON FREEWAY WITH INCREASED SPEED LIMIT
}

\author{
Lu, Q. \& Tettamanti, T. \\ Department of Control for Transportation and Vehicle Systems, \\ Budapest University of Technology and Economics, Budapest, Hungary \\ E-Mail: qiong.lu@edu.bme.hu, tettamanti.tamas@kjk.bme.hu
}

\begin{abstract}
As a revolutionary technology, Connected and Automated Vehicles (CAVs) have great potential to reduce traffic collisions, increase transportation system performance, and improve environmental sustainability. This research aims to investigate the benefit or trade-off of CAVs on freeway capability, fuel consumption, and emission. To fulfil this goal, the open-source traffic simulation software, SUMO was used to model CAV fleets. This study conducted a sensitivity analysis on three variables, i.e., CAV penetration, CAV time headway, and freeway speed limit. As expected, the simulation results justified that the shorter the time headway is, the higher maximum flow can be achieved. Higher speed limit contributes to a faster free-flow speed and a more substantial road capacity. While at the same time, it weakens the stability of the flow. From the perspective of fuel economy and environment, a recommended optimal time headway must be found.

(Received in December 2020, accepted in March 2021. This paper was with the authors 1 week for 2 revisions.)
\end{abstract}

Key Words: CAV Penetration, Time Headway, Speed Limit, Freeway Capacity, Fuel Consumption, Emission

\section{INTRODUCTION}

Most current activities in intelligent transport systems (ITS) have centred on the technologies and applications of Connected and Automated Vehicles (CAVs). CAV is a combination definition of Connected Vehicle (CV) and Automated Vehicle (AV). It is a revolutionary technology with great potential to reduce traffic collisions, increase transportation system performance, and improve life quality. The Society of Automotive Engineering (SAE) classifies AVs into six levels of driving automation according to the dynamic driving tasks performed by the vehicle operating automation systems [1]. CAV is a vehicle with the ability to communicate bi-directionally with other cars and infrastructures. CAVs connect through the vehicle to vehicle (V2V) and vehicle to infrastructure (V2I) communication in a collaborative and meaningful manner. The infrastructure is a centralized traffic management centre. The comprehensive and timely information improves drivers' situational awareness and enhances the safety and efficiency of operating vehicles.

Cooperative Adaptive Cruise Control (CACC) and adaptive cruise control (ACC) are essential technologies towards fully automated driving. They can be used to simulate the cruising behaviour of AVs and CAVs respectively to study how AVs/CAVs can beneficially impact transportation systems [2]. At present, there is no consensus on the right value for the headway of self-driving cars. By comparing the empirical data from simulations, Young and Stanton found a striking increase in the reaction time under automated condition compared with the manual driving vehicles [3]. They claimed that the automated vehicle drivers were less anticipative of making an emergency reaction than driving manually. CAV developers confronted a dilemma about the proper setting of safe time headway based on the driver's response times $[4,5]$. Oliveira et al. investigated how the driving behaviours of autonomous vehicles influence trust and acceptance [6]. Olia et al. and Abdulsattar et al. simulated the CAVs with a time headway of 0.5 seconds $[7,8]$. While Berrazouane et al. used a large time headway (even large than the conventional vehicles) to model an AV behaviour demonstrating that the 
preferred driving behaviour of AVs is still uncertain [9]. Therefore, it is important to do a sensitivity analysis on time headway. which is one of the aims of our paper.

The speed limit of freeways varies from country to country. The speed limit has strong impacts on the road network performance, especially when the times of CAVs arrives. Ye and Yamamoto concluded that placing a higher speed limit for CAVs on the dedicated lane can improve the performance of CAV specific lane [10]. Besides, the variable speed limit (VSL) warning is an important technology to have better speed management under adverse weather conditions on freeway [11]. Chen et al. proposed a speed guidance control which can improve the efficiency and safety of traffic flow on the urban expressway [12]. With the improvement of the safety performance of self-driving cars, it should become a trend to increase the freeway speed limit. Therefore, studying the effect of different speed limits on CAV performance is meaningful.

Various researchers examined the impacts of microscopic driving behaviour in AVs or CAVs with the help of simulation as system dynamics simulation plays an essential role in transportation planning [13]. Kavicka et al. proposed an agent-based architecture for simulating the transportation system [14]. The car following parameters (e.g., headway, speed, penetration rate) and their effects on road capacity were studied. In general, a shorter headway could boost maximum throughput. Contrarily, an increasing time headway would lead to a decreasing road capacity. Lower speed vehicles could contribute to the stability of vehicle flows. However, as a consequence, they would cut down the bottleneck's capacity. What's more, the delay and travel time would increase at the same time [15-17]. Some of these studies revealed that AVs penetration rate in the intermediate (or even lower) range could result in a significant capacity upgrade [15]. Talebpour et al. found the throughput and trip time stability were improved considerably when the AVs penetration rate surpassed $30 \%$ when AVs had a dedicated lane on a four-lane freeway [16]. However, road capability increased marginally until the CACC ratio surpassed 85 percent, according to Tientrakool et al. [18]. Lu et al. investigated the impacts of CAVs on the urban road network by simulation on grid network and real-world city road network. They found a $16 \%$ improvement on road capacity with $100 \%$ automated vehicle scenarios [19]. Olia et al. found that the situation with the $100 \%$ penetration of cooperative vehicles could boost the highway capacity by $300 \%$ [7].

Autonomous vehicles have two opposing effects on the environment. On the one hand, good driving technology would reduce fuel consumption and exhaust emissions. On the other hand, increased transportation demand would increase pollution in transportation [20]. The primary aim of CAV technology is to improve traffic safety and make available better mobility services. Though, CAV would predictably and significantly transform the transportation sector environmental profile as well. Cao and Zöldy evaluated the impact of CAV driving behaviour in a roundabout on fuel consumption and emission reductions [21]. They summarized that driving behaviour, effective of automation systems, roundabout physical parameters as well as vehicle types played important roles in energy usage. Taiebat et al. classified the impacts of $\mathrm{CAVs}$ on the environment into four levels, which are vehicle, transportation system, urban system, and society levels. As for the vehicle level, operation, electrification, design, and platooning could affect the vehicles' consumption and emission. This research focused on the impacts of the vehicle behaviour. Greater driving efficiency might be obtained with CAVs through an assortment of mechanisms, like, counting optimum driving cycle, dynamic system optimum routing, less idling, reducing cold starts, and speed harmonization [22].

Fewer idling or less cold start could help decrease fuel consumption and reduce emissions. Cold starts are significant causation of some air pollutants from the transportation area, for instance, NOx, CO, and volatile organic compounds (VOCs) [23]. A few properties of CAVs might lead to more energy waste. Radars, sensors, information exchanges, and high-speed network connections need more auxiliary energy from cars, which exposes as extra noteworthy 
power draw and subsequently more fuel consumption. Power used in sensing, connectivity units, and computing accessories could considerably change the CAVs energy efficiency. Moreover, advanced protection in CAVs might bring about higher freeway speeds. Because aerodynamic drag forces build up quadratically with speed, therefore, higher speeds bring about higher energy waste above a certain threshold. For example, a velocity increasing from 70 to 80 miles per hour was announced to raise average power usage by $13.9 \%$ per mile [24]. Even though, it is convincing that improved security in CAVs can empower relaxation of speed limits for freeways. In most countries vehicles are currently restricted to below optimal speeds, which lead to some fuel conservations. This aspect gains less consideration in the literature.

The contribution of our paper is twofold. First, the shorter the time headway is, the higher the maximum flow becomes. Reducing time headway in a certain range may result in lower fuel consumption and emission. When the time headway lower than this range, the energy consumption and emission increase sharply. From the perspective of fuel economy and environment, a recommended optimal time headway must be found. Second, a higher speed limit contributes to a faster free-flow speed and a more substantial road capacity. While at the same time, it weakens the stability of the flow. The speed limit differs from country to country. There are few papers investigate the CAV under different speed limits scenarios. This paper provides an insight into the impacts of varying speed limits when it comes to CAVs.

The following is the outline of the paper. The theoretical preliminaries on the fundamental diagram and the macroscopic variable calculate process are presented in Section 2. The SUMO simulator environments, the AVs modelling technique, and the fundamental curve fitting process are all discussed in Section 3. The core outcomes of the simulation experiment are discussed in Section 4. Section 5 concludes with some closing remarks.

\section{THEORETICAL FOUNDATIONS: MACROSCOPIC TRAFFIC FLOW MODELLING OF FREEWAY TRAFFIC}

The fundamental diagram is a well-acknowledged theory to characterize freeway traffic dynamics in a macroscopic way. In this approach averaged traffic flow variables are considered on a given road stretch, i.e., traffic density $k$ (vehicles $/ \mathrm{km})$, space mean speed $V(k)(\mathrm{km} / \mathrm{h})$ depending on $k$, as well as traffic flow $Q(V(k))$ (vehicles/h). The fundamental diagram of traffic flow is selected to show the impacts on the freeway capability. The theory of fundamental diagram includes three important relationships among the macroscopic variables, which are flow-density, speed-density, and speed-flow. These three diagrams are related via the following equation adopted from fluid dynamics.

$$
Q(V(k))=V(k) \cdot k
$$

A wide range of diverse forms of fundamental diagrams are available in the literature [25]. One practical form of the fundamental diagrams (which well reflects the real-world observations on freeways) is proposed by Newell [26], i.e., the triangular flow-density relationship.

$$
Q=\min \left\{v \cdot k ; w \cdot\left(k_{j}-k\right)\right\}, \text { for } 0 \leq k \leq k_{j}
$$

where $v$ is free-flow speed, $k$ is the vehicle density, $w$ means the backward wave speed, as well as $k_{j}$ stands for the jam density. The freeway link, in this paper, is assumed to follow the kinematic wave theory with all lanes having the same free-flow speed $v$ and the same backward wave speed $w$, see Fig. 1. $Q_{m}$ is the maximum flow rate, and the corresponding density $k_{c}$ is referred to as the critical density. From the other parameters, $k_{j}=Q_{m}(1 / v+1 / w)$ or $k_{j}=1000 /\left(l_{v}\right.$ $+\min (a p)$, the jam density can be estimated [27]. Here, $l_{v}$ is the length of vehicle, and minGap is the minimum distance between two consecutive vehicles. Within the triangular flow-density relationship, the proportion of jam density $k_{j}$ to critical density $k_{c}$ is $1+v / w$, as shown in Eq. (3). 


$$
\frac{k_{j}}{k_{c}}=1+\frac{v}{w}
$$

The maximum flow $Q_{m}$ is derived as follows.

$$
Q_{m}=\frac{k_{j} w v}{w+v}
$$

In this study, the simulated traffic flow is in line with the kinematic wave theory and the triangular fundamental diagram (FD) is applied. Cassidy et al. had verified that the triangular fundamental diagram is applied to fit the data collected from the simulations on the freeway test network [28].

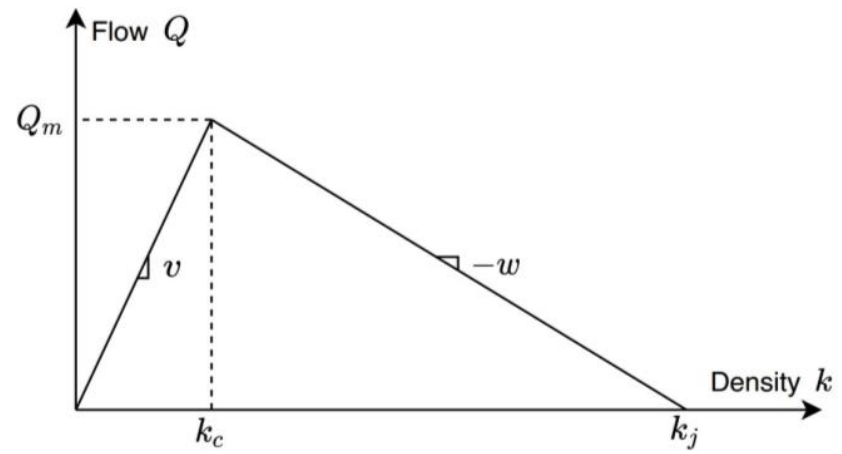

Figure 1: Triangular fundamental diagram.

\section{SIMULATION BASED ANALYSIS TO ASSESS THE IMPACT OF CAVS ON THE FUNDAMENTAL DIAGRAM}

For realistic simulation based analysis an open-source, microscopic traffic simulation software, the Simulation of Urban Mobility (SUMO), was applied. In a SUMO simulation, each car has its route, and moves individually through the road network, with a variety of options for introducing randomness. SUMO allows users to modify and control a wide range of parameters concerning the cars, the road network, and the simulation during run time with its flexible Matlab interface - TraCI4Matlab.

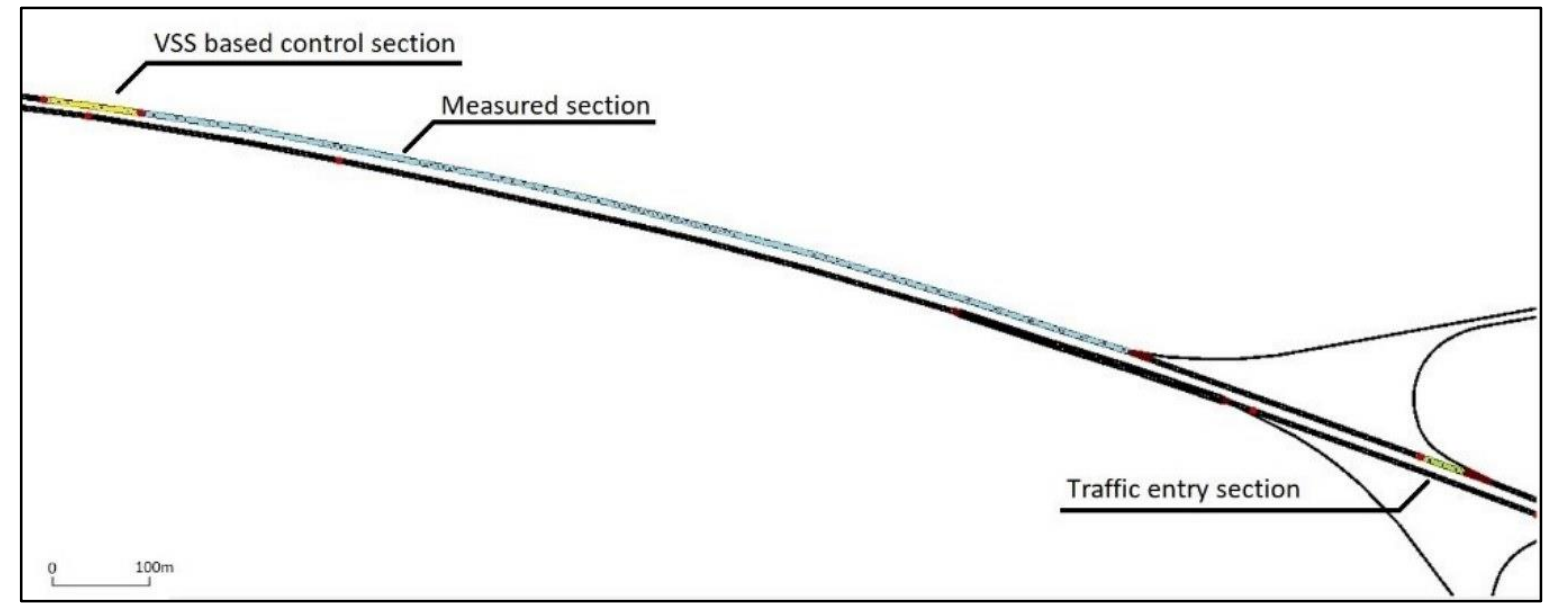

Figure 2: The simulated traffic network.

As shown in Fig. 2, a real-world network was selected for simulation based analysis. The test network is a section of the European designation E60 (Hungarian highway M1) near Herceghalom city. The base data for geometry, traffic flow, as well other road features were gathered directly from the open database of Hungarian Public Roads. 
In this research, a sensitivity analysis was carried out investigating three parameters: time headway, CAV penetration, and the speed limits to find their impacts on the FD, the throughput of the highway, and the energy consumption and air pollution of vehicles.

The realization of CAV was accomplished through the parameterization of the vehicle behavioural models which are the Krauss car-following model and the LC2013 lane-changing models. The execution of different type of cars into SUMO simulation was realized by different vehicle types definition (declaring sets of cars with the same parameters and performance in transportation system).

In this work, 2 vehicle types were applied, which are human driven vehicles (HDV), and CAV. The parameters are listed in Table I.

Table I: The constant attributes of the different vehicle types.

\begin{tabular}{|c|c|c|c|c|c|c|}
\hline $\begin{array}{c}\text { Vehicle } \\
\text { type }\end{array}$ & $\begin{array}{c}\text { Acceleration } \\
\left(\mathrm{m} / \mathrm{s}^{2}\right)\end{array}$ & $\begin{array}{c}\text { Deceleration } \\
\left(\mathrm{m} / \mathrm{s}^{2}\right)\end{array}$ & $\begin{array}{c}\text { Speed limit } \\
(\mathrm{km} / \mathrm{h})\end{array}$ & $\boldsymbol{\sigma}$ & $\boldsymbol{\tau}(\mathrm{s})$ & Speed factor \\
\hline HDV & 2.6 & 4.5 & $\begin{array}{c}80-200 \mathrm{with} \\
\text { an increment } \\
\text { of } 20 \mathrm{~km} / \mathrm{h}\end{array}$ & 0.5 & 2 & $\operatorname{normc}(1,0.1,0.2,2)$ \\
\hline CAV & 2.6 & 4.5 & $\begin{array}{c}80-200 \mathrm{with} \\
\text { an increment } \\
\text { of } 20 \mathrm{~km} / \mathrm{h}\end{array}$ & 0 & $\begin{array}{c}0.1-1.9 \text { with } \\
\text { a } 0.2 \mathrm{~s} \\
\text { increment }\end{array}$ & normc $(1,0.1,0.2,2)$ \\
\hline
\end{tabular}

The acceleration and deceleration are considered as constant for human driven vehicle (HDV) and connected automated vehicle (CAV). Speed limit means the speed limit of freeway for both HDV and CAV. It varies from $80 \mathrm{~km} / \mathrm{h}$ to $200 \mathrm{~km} / \mathrm{h}$ with an increment of $20 \mathrm{~km} / \mathrm{h}$. Theoretical high speed limits are investigated. As the technologies are developing, a higher speed limit may be possible in the future. The $\sigma$ in SUMO is the driver imperfection for which the value 0 denotes perfect driving. The CAV is assumed have the perfect driving behaviours. The $\tau$ is the minimum desired time headway of drivers. The time headway for HDVs is a constant that equals to 2 seconds. The time headway for CAVs vary from $0.1 \mathrm{~s}$ to $1.9 \mathrm{~s}$ with a $0.2 \mathrm{~s}$ increment. The speed factor is used to model the desired driving speed variation. It represents the driver's attitude towards the road's speed limit, by introducing a randomly generated multiplier. The speed factor in this paper is a normal distribution which is given as "normc(mean, deviation, minimum, maximum)" form. Using speed factor equals to "normc(1, $0.1,0.2,2)$ " brings a velocity distribution where $95 \%$ of the cars driven between 0.8 to 1.2 times of the allowed speed limit.

A full fundamental diagram (FD) should be built at each observed speed limit to realise the intentions stated at the start of this part. Relevant data were collected from the measured section in Fig. 2. The collected data are mainly the volume of vehicles on the measured segment as well as their average velocity. The traffic flow increased up continuously till the maximum road network capacity. Then an outside factor was applied to destabilise the flow. After multiple tests, the Variable Speed Signs (VSS) based traffic flow interference was chosen as it was the most reliable way to simulate full FDs. The length of the measured edge is $1033.12 \mathrm{~m}$, and it has two lanes, as shown in Fig. 2. Data is being collected every ten simulated seconds and was valid for the given time step ( 0.1 seconds). The nominal traffic flow was set based on the Hungarian Public Road's real world measurement.

In order to obtain a full FD, the simulation began with a low flow rate of traffic. The cars were introduced with Poisson distribution to guarantee randomization of the simulations. After planned periods, the vehicle flow increased up until reached the network's peak capacity. And then, the determined flow control program, VSS, was triggered to generate growing amounts of barrier on the road network, until the simulation reached a near grid-lock state. At this time, the interference terminated, and the flow started to release. When the jammed traffic flow 
disappeared, this simulation completed. Subsequently, a new simulation began with higher CAV penetration. Fig. 3 shows the automatized simulation process of the work. The simulation was operated under three levels of nested loops. The variables of three-level loops from inside to outside are time headway $(\tau)$, CAV penetration (pene), and speed limit $(S L)$. The time headway of CAV starts at $1.9 \mathrm{~s}$ and goes down to $0.1 \mathrm{~s}$ with a $0.2 \mathrm{~s}$ step length. The middle loop changes CAV penetration rates starting from $0 \%$ to $100 \%$ with a $10 \%$ increment. The outer loop defines the speed limit of the simulation, starting from $80 \mathrm{~km} / \mathrm{h}$ with an increment of 20 $\mathrm{km} / \mathrm{h}$ to $200 \mathrm{~km} / \mathrm{h}$. The simulation started with the scenario where the speed limit equals 80 $\mathrm{km} / \mathrm{h}$, CAV penetration is $0 \%$, and time headway equals $1.9 \mathrm{~s}$. After different time headway scenarios simulated, the simulation goes on with the next predefined CAV penetration and reset the time headway to be $1.9 \mathrm{~s}$. After running a full penetration range, the simulation goes on with a higher speed limit. The CAV penetration is reset to 0 ; time headway to $1.9 \mathrm{~s}$. This procedure repeats until the outer loop finishes, which means all scenarios are simulated.

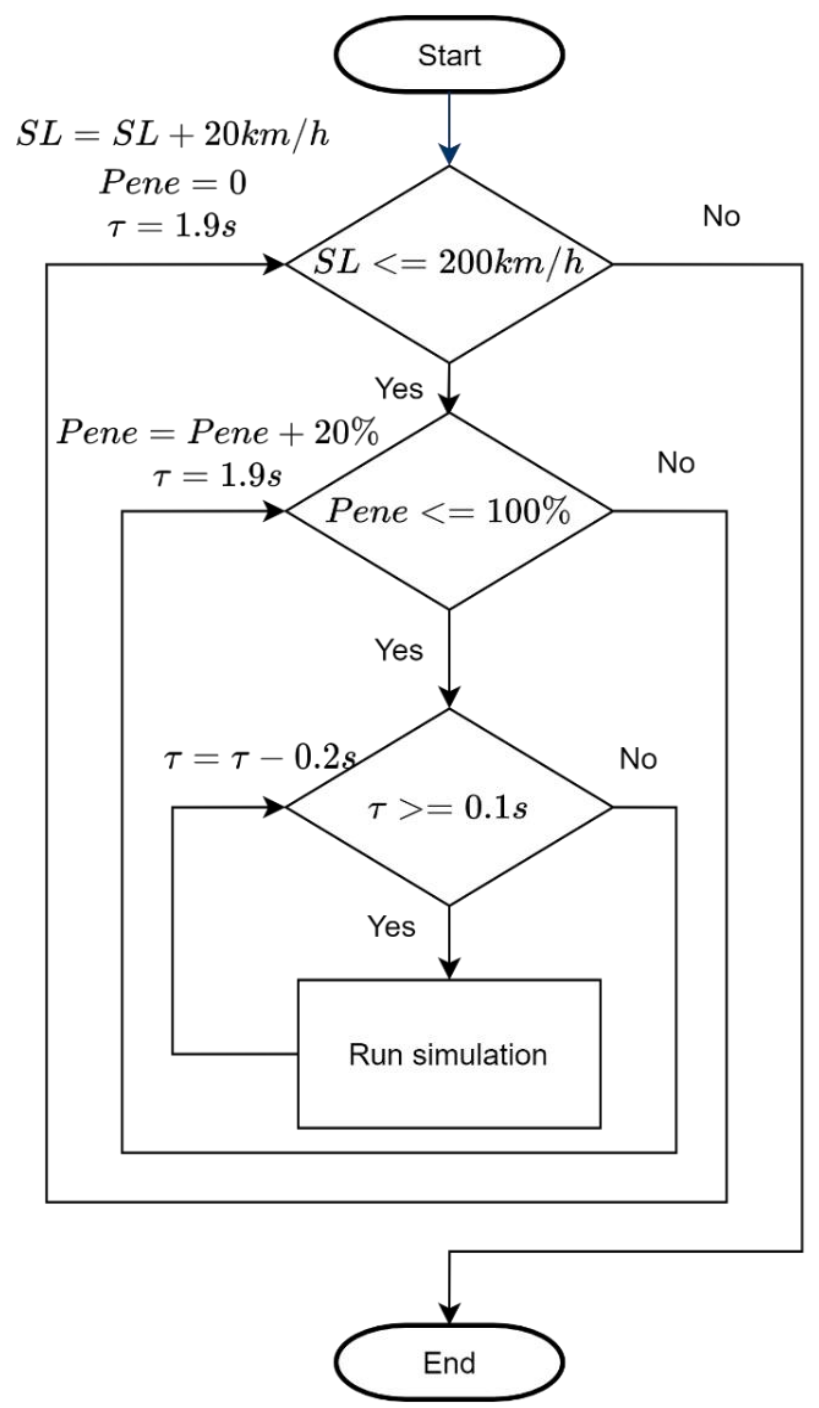

Figure 3: The automatized simulation process.

\section{SIMULATION RESULTS}

To qualify the impacts of CAV penetration, reaction time variation, and speed limits, different simulation scenarios were carried out. The freeway capability and environment impacts were investigated. 


\subsection{Impacts of CAVs on freeway capacity}

The general aim of this research is to do the sensitivity analysis on time headway, CAV penetration, and the speed limits to investigate their impacts on the freeway capability and the fuel consumption and emissions of vehicles. In this paper, the fundamental diagram is used to estimate the capacity of freeways.
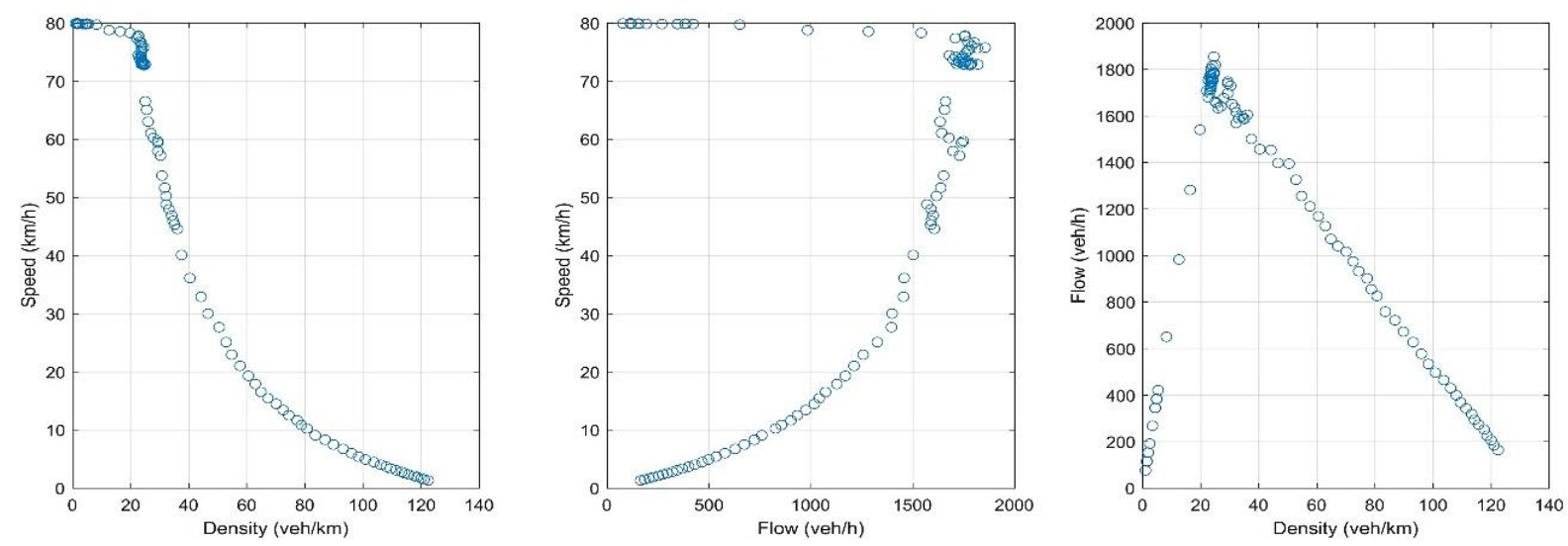

Figure 4: Fundamental diagram of a simulation (penetration $=40 \%, \tau=1.1 \mathrm{~s}$, speed limit $=80 \mathrm{~km} / \mathrm{h}$ ).

Fig. 4 shows the fundamental diagram of one of the simulations when the CAV penetration is $40 \%$, CAV time headway is $1.1 \mathrm{~s}$, and the speed limit is $80 \mathrm{~km} / \mathrm{h}$. They show the fundamental diagram's typical shape, shared between all other simulation scenarios. From the flow-density relationship, one can see that the simulations have a triangular fundamental diagram. Therefore, a triangular fundamental diagram model was chosen to fit the collected data. The capacity of an edge is defined by the maximum vehicle number that passes the network per hour. It can be defined as the product of the traffic density and the state mean speed [29]. In this paper, the maximum traffic flow is calculated to indicate the practical capacity of the road.

Fig. 5 depicts the flow-density relationship of the simulations when the speed limit is $80 \mathrm{~km} / \mathrm{h}$ and CAV penetration is $60 \%$.

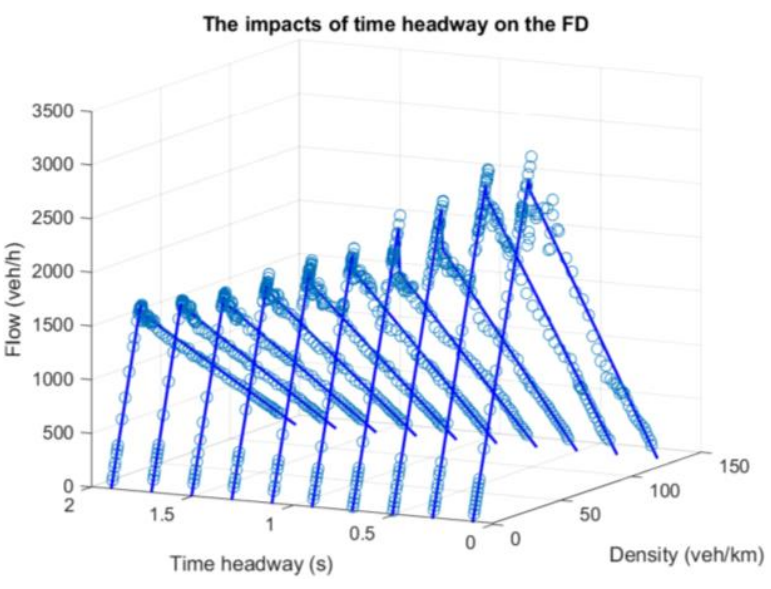

Figure 5: Flow-Density relationship changes with time headway.

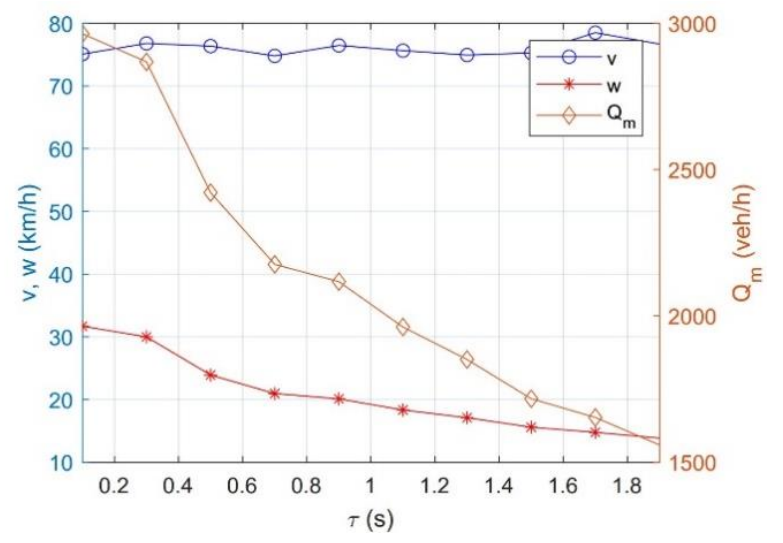

Figure 6: The triangular fundamental diagram parameters change with the time headway.

The time headway of CAV varies from $1.9 \mathrm{~s}$ to $0.1 \mathrm{~s}$. It is obvious that the maximum flow is increasing with the decrease of time headway. As the time headway decreases, the points at the maximum flow rate become more and more scattered. Therefore, reducing the time headway is a double-edged sword. On the one hand, it increases the capacity of the road, on the other hand, it reduces the stability of the traffic flow. 
Fig. 6 reveals the values of the fitted fundamental diagram. The free flow speed $v$ is relatively stable. The wave speed $w$ increases substantially with a shorter time headway. The maximum flow volume increases when the time headway becomes shorter.

Fig. 7 shows the impacts of CAV penetration rate on the flow-density relationship when the speed limit is $80 \mathrm{~km} / \mathrm{h}$ and time headway is 1.1 seconds.

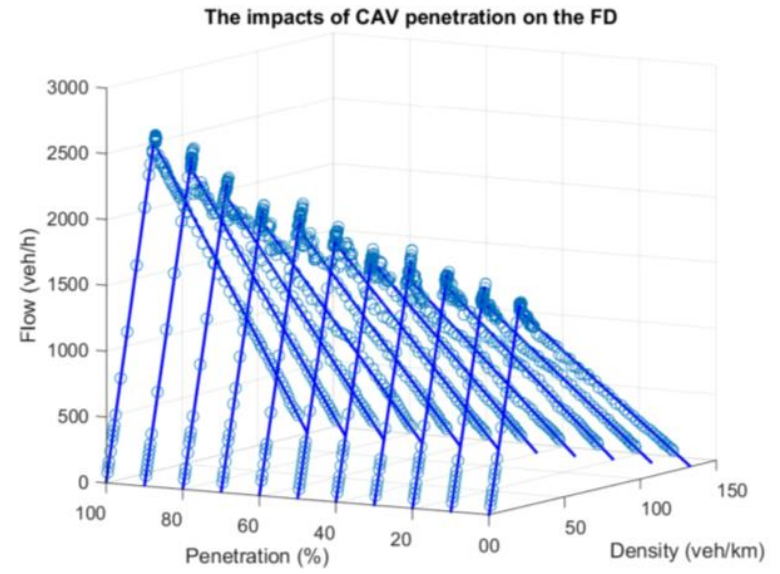

Figure 7: Flow-Density relationship changes with CAV penetration.

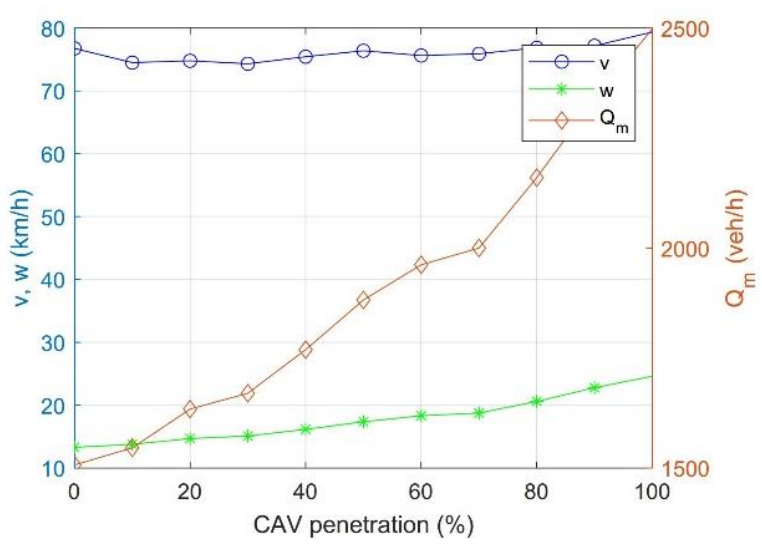

Figure 8: The triangular fundamental diagram parameters change with the CAV penetration.

Obviously, with the gradual increase in the CAV penetration, the maximum flow is also rapidly augmenting. However, when the penetration rate is less than $40 \%$, the advantage is not so apparent. This observation is in line with the results of $[17,30]$. The highest traffic flow of pure CAV scenario is almost twice that of pure traditional vehicle scenario. Fig. 8 depicts the changes of fitted triangular fundamental diagram parameters. The free flow speed $v$ almost keeps the same. On the contrary, the wave speed $w$ rises sharply. The maximum flow also increases drastically when there are more and more CAVs on the road.

Fig. 9 reveals the variation of the observed maximum traffic flow with CAV penetration and time headway. From the impact of traffic flow, the shorter the time headway and the higher the market penetration is, the better the road performance becomes.

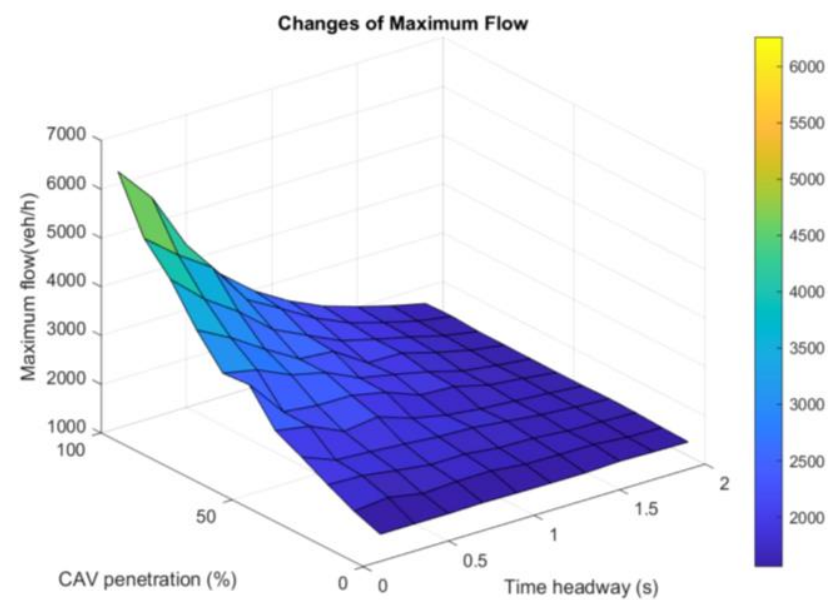

Figure 9: Maximum flow changes.

Table II shows the observed maximum flow volume changes with different CAV penetration and time headway. The unit of the maximum flow is veh/h. The simulations with $0 \%$ of CAV penetration rate are treated as the baseline in this research. As the CAV penetration rises, the maximum flow volume grows as well. The shorter the time headway is, the higher the growth is. 
Table II: Maximum flow changes along with the CAV penetration and time headway.

\begin{tabular}{|c|c|c|c|c|c|c|c|c|c|c|}
\hline Penetration & $\mathbf{1 . 9} \mathbf{s}$ & $\mathbf{1 . 7} \mathbf{s}$ & $\mathbf{1 . 5} \mathbf{s}$ & $\mathbf{1 . 3} \mathbf{s}$ & $\mathbf{1 . 1} \mathbf{s}$ & $\mathbf{0 . 9} \mathbf{s}$ & $\mathbf{0 . 7} \mathbf{s}$ & $\mathbf{0 . 5} \mathbf{s}$ & $\mathbf{0 . 3} \mathbf{s}$ & $\mathbf{0 . 1} \mathbf{~}$ \\
\hline $\mathbf{0 \%}$ & 1559 & 1570 & 1587 & 1558 & 1562 & 1567 & 1580 & 1566 & 1566 & 1565 \\
\hline $\mathbf{1 0} \%$ & 1580 & 1593 & 1656 & 1657 & 1672 & 1746 & 1740 & 1702 & 1833 & 1763 \\
\hline $\mathbf{2 0} \%$ & 1609 & 1640 & 1659 & 1718 & 1733 & 1814 & 1809 & 1902 & 2005 & 2029 \\
\hline $\mathbf{3 0} \%$ & 1622 & 1663 & 1713 & 1793 & 1871 & 1896 & 1957 & 2011 & 2053 & 2323 \\
\hline $\mathbf{4 0} \%$ & 1610 & 1672 & 1724 & 1773 & 1855 & 2004 & 2241 & 2153 & 2470 & 2589 \\
\hline $\mathbf{5 0} \%$ & 1611 & 1700 & 1774 & 1954 & 1991 & 2258 & 2406 & 2413 & 2492 & 3259 \\
\hline $\mathbf{6 0} \%$ & 1615 & 1693 & 1789 & 1984 & 2138 & 2279 & 2600 & 2762 & 3075 & 3218 \\
\hline $\mathbf{7 0} \%$ & 1623 & 1731 & 1894 & 2092 & 2103 & 2549 & 2606 & 3016 & 3458 & 3831 \\
\hline $\mathbf{8 0} \%$ & 1627 & 1754 & 1935 & 2102 & 2282 & 2604 & 2936 & 3510 & 3978 & 4596 \\
\hline $\mathbf{9 0} \%$ & 1663 & 1780 & 1962 & 2163 & 2471 & 2877 & 3273 & 4144 & 4606 & 5160 \\
\hline $\mathbf{1 0 0} \%$ & 1650 & 1817 & 2008 & 2231 & 2548 & 2960 & 3519 & 4331 & 5506 & 6257 \\
\hline
\end{tabular}

The speed limit has a direct impact on the free flow speed. With a higher speed limit, the value of free flow speed $v$ is expected to increase. As a representative example, consider Fig. 10 and Table II which display the simulation results with $1.1 \mathrm{~s}$ time headway and $50 \%$ penetration rate for CAVs. In Fig. 10, the scatter points represent the collected data from the simulations. The solid lines are the fitted triangular fundamental diagrams. Fig. 10 reveals the free flow speed and road capacity increase when the speed limit gets higher. When the speed limit is higher than $160 \mathrm{~km} / \mathrm{h}$, the measured points around the critical density are more scattered. Therefore, in order to maintain the stability of the flow around the critical density, the speed limit should not exceed $160 \mathrm{~km} / \mathrm{h}$.

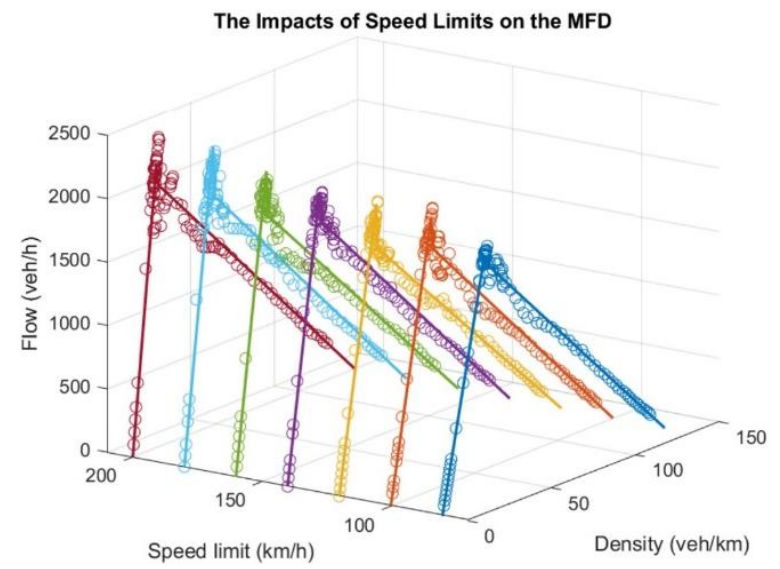

Figure 10: The impacts of speed limit on fundamental diagram.

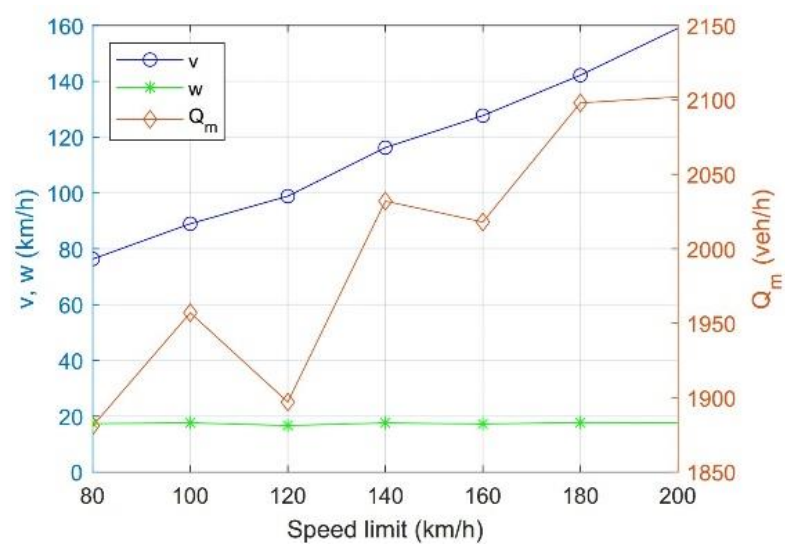

Figure 11: The triangular fundamental diagram parameters change with the speed limit.

As shown in Fig. 11, the free flow speed $v$ increases with a higher speed limit. However, the value of $w$, the backward wave speed, remains almost the same while the speed limit varies. From Eq. (3), it is easy to derive that the critical density $k_{c}$ moves to the left hand side slightly when the speed limit increases. This is consistent with the result observable in Fig. 10 where the maximum traffic flow shows a growing trend, but the maximum difference is only $284 \mathrm{veh} / \mathrm{h}$.

\subsection{Impacts of CAVs on fuel consumption and emissions}

In the simulations, it is assumed that the travel demand remains the same when CAV penetration increases. The aggregate fuel consumption and $\mathrm{CO}_{2}$ during a whole simulation are investigated. The emission model used in the simulation was HBEFA3. For simplicity, the emission class for all vehicles was gasoline driven passenger car of European emission standards 5. Fig. 12 indicates the aggregate fuel consumption over the entire simulation when the speed limit is 
$80 \mathrm{~km} / \mathrm{h}$. When the CAV time headway is 1.1 seconds, the consumption does not change much, no matter what the CAV penetration rate is.

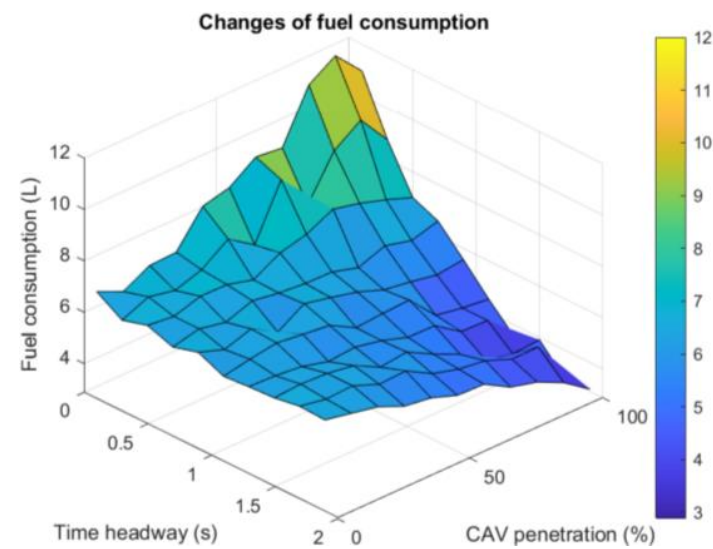

Figure 12: Fuel consumption changes.

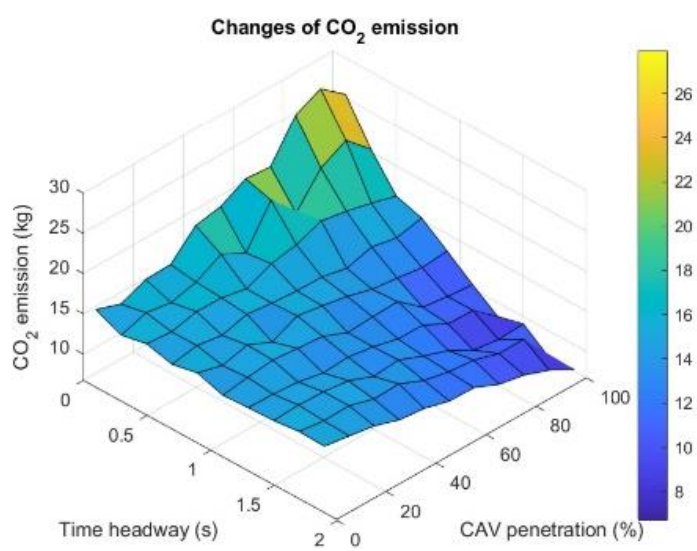

Figure 13: $\mathrm{CO}_{2}$ emission changes.

However, when the time headway less than 0.5 seconds, fuel consumption increases sharply with the rise in CAV penetration. The all HDV scenarios serve as the baseline. From Fig. 12, one can observe that when time headway is longer than 0.5 seconds the aggregate fuel consumption drops with the increase of CAV penetration. When the time headway becomes shorter than 0.5 seconds, there will be more frequent braking and starting and more changes in velocity, especially in traffic jams. As mentioned in the introduction, breaks, starts, and velocity variations may contribute to increasing fuel consumption. From the perspective of reducing fuel consumption, 0.5 seconds is the lower recommended limit of the time headway setting for CAVs.

The results of $\mathrm{CO}_{2}$ emission share a similar shape with the fuel consumption. Fig. 13 indicates the aggregate $\mathrm{CO}_{2}$ emission during the whole simulation. When the CAV time headway is under 1.1 seconds, the emission does not change much no matter what the CAV penetration rate is. However, when the time headway is less than 0.5 seconds, carbon dioxide emissions increase sharply with the increase of CAV market share. The all HDV scenarios serve as the baseline. From Fig. 13, one can see that when time headway is longer than 0.5 seconds the aggregate $\mathrm{CO}_{2}$ emission is dropping with the increase of $\mathrm{CAV}$ penetration. From the perspective of reducing carbon dioxide emissions, 0.5 seconds is the lower limit of the time headway setting for CAVs when speed limit is $80 \mathrm{~km} / \mathrm{h}$.

\section{CONCLUSION}

This paper carried out a sensitivity analysis on time headway, CAV penetration, as well as the speed limits. The impacts on freeway traffic flow, fuel consumption, and emission have been investigated. As shown in the result section, the free flow speed $v$ is relatively stable when CAVs penetration and time headway changes. The wave speed $w$ increases substantially when the time headway becomes shorter. Furthermore, it can be concluded that the shorter the time headway is, the higher the maximum flow becomes. At the same time, fuel consumption drops with the increase of CAV penetration when the CAV time headway is longer than 0.5 seconds when speed limit is $80 \mathrm{~km} / \mathrm{h}$. On the contrary, when time headway is shorter than $0.5 \mathrm{~s}$, fuel consumption and $\mathrm{CO}_{2}$ emission increase sharply while $\mathrm{CAV}$ penetration increases. Therefore, reducing time headway in a certain range may result in lower fuel consumption and emission. When the time headway lower than this range, the energy consumption and emission increase sharply. Consequently, a recommended optimal time headway should be found to balance the CAVs' impacts on the road capacity and fuel consumption. The speed limit directly influences the free-flow speed. As speed limit rises, free-flow speed and road capacity do the same as well. 
As shown in the result section, the maximum flow increases with the enlarged speed limit in the analysed scenario. However, when the speed limit is higher than $160 \mathrm{~km} / \mathrm{h}$, the measured points around the critical density are more scattered around with the speed limit's rise. A higher speed limit contributes to a faster free-flow speed and a more substantial road capacity. At the same time, it weakens the stability of the flow. In the future, with the optimization of CAV performance and increasing the speed limit might be an effective method to improve the expressway capacity.

\section{ACKNOWLEDGEMENT}

The research was supported by the Hungarian Government and co-financed by the European Social Fund through the project "Talent management in autonomous vehicle control technologies" (EFOP3.6.3-VEKOP-16-2017-00001). The research was also supported by the fund of the National Research, Development and Innovation Office Hungary (2019-2.1.7-ERA-NET-2021-00019).

\section{REFERENCES}

[1] SAE International - SAE On-Road Automated Vehicle Standards Committee (2018). Taxonomy and Definitions for Terms Related to Driving Automation Systems for On-Road Motor Vehicles, J3016 Standard, SAE International, Warrendale, doi:10.4271/J3016 201806

[2] Makridis, M.; Mattas, K.; Ciuffo, B.; Alonso Raposo, M.; Toledo, T.; Thiel, C. (2018). Connected and automated vehicles on a freeway scenario: Effect on traffic congestion and network capacity, Proceedings of $7^{\text {th }}$ Transport Research Arena TRA 2018, 10 pages, doi:10.5281/zenodo.1483132

[3] Young, M. S.; Stanton, N. A. (2007). Back to the future: Brake reaction times for manual and automated vehicles, Ergonomics, Vol. 50, No. 1, 46-58, doi:10.1080/00140130600980789

[4] Taieb-Maimon, M.; Shinar, D. (2001). Minimum and comfortable driving headways: Reality versus perception, Human Factors: The Journal of the Human Factors and Ergonomics Society, Vol. 43, No. 1, 159-172, doi:10.1518/001872001775992543

[5] Goodrich, M. A.; Boer, E. R. (2003). Model-based human-centered task automation: a case study in ACC system design, IEEE Transactions on Systems, Man, and Cybernetics - Part A: Systems and Humans, Vol. 33, No. 3, 325-336, doi:10.1109/TSMCA.2003.817040

[6] Oliveira, L.; Proctor, K.; Burns, C. G.; Birrell, S. (2019). Driving style: How should an automated vehicle behave?, Information, Vol. 10, No. 6, Paper 219, 20 pages, doi:10.3390/info10060219

[7] Olia, A.; Razavi, S.; Abdulhai, B.; Abdelgawad, H. (2017). Traffic capacity implications of automated vehicles mixed with regular vehicles, Journal of Intelligent Transportation Systems, Vol. 22, No. 3, 244-262, doi:10.1080/15472450.2017.1404680

[8] Abdulsattar, H.; Rayeedul Kalam Siam, M.; Wang, H. (2020). Characterisation of the impacts of autonomous driving on highway capacity in a mixed traffic environment: an agent-based approach, IET Intelligent Transport Systems, Vol. 14, No. 9, 1132-1141, doi:10.1049/iet-its.2019.0285

[9] Berrazouane, M.; Tong, K.; Solmaz, S.; Kiers, M.; Erhart, J. (2019). Analysis and initial observations on varying penetration rates of automated vehicles in mixed traffic flow utilizing SUMO, 2019 IEEE International Conference on Connected Vehicles and Expo, 7 pages, doi:10.1109/ICCVE45908.2019.8965065

[10] Ye, L.; Yamamoto, T. (2018). Impact of dedicated lanes for connected and autonomous vehicle on traffic flow throughput, Physica A: Statistical Mechanics and its Applications, Vol. 512, 588-597, doi:10.1016/j.physa.2018.08.083

[11] Yang, G.; Ahmed, M. M.; Gaweesh, S. (2019). Impact of variable speed limit in a connected vehicle environment on truck driver behavior under adverse weather conditions: driving simulator study, Transportation Research Record: Journal of the Transportation Research Board, Vol. 2673, No. 7, 132-142, doi:10.1177/0361198119842111

[12] Chen, D. S.; Yu, X. X.; Hu, K. Q.; Sun, X.; Xia, Y. Y. (2014). Safety-oriented speed guidance of urban expressway under model predictive control, International Journal of Simulation Modelling, Vol. 13, No. 2, 219-229, doi:10.2507/IJSIMM13(2)CO8 
[13] Suryani, E.; Hendrawan, R. A.; Adipraja, P. F. E.; Indraswari, R. (2020). System dynamics simulation model for urban transportation planning: a case study, International Journal of Simulation Modelling, Vol. 19, No. 1, 5-16, doi:10.2507/IJSIMM19-1-493

[14] Kavicka, A.; Klima, V.; Adamko, N. (2007). Simulations of transportation logistic systems utilising agent-based architecture, International Journal of Simulation Modelling, Vol. 6, No. 1, 13-24, doi:10.2507/IJSIMM06(1)2.075

[15] Jerath, K.; Brennan, S. N. (2012). Analytical prediction of self-organized traffic jams as a function of increasing ACC penetration, IEEE Transactions on Intelligent Transportation Systems, Vol. 13, No. 4, 1782-1791, doi:10.1109/tits.2012.2217742

[16] Talebpour, A.; Mahmassani, H. S.; Elfar, A. (2017). Investigating the effects of reserved lanes for autonomous vehicles on congestion and travel time reliability, Transportation Research Record: Journal of the Transportation Research Board, Vol. 2622, No. 1, 1-12, doi:10.3141/2622-01

[17] Van Arem, B.; van Driel, C. J. G.; Visser, R. (2006). The impact of cooperative adaptive cruise control on traffic-flow characteristics, IEEE Transactions on Intelligent Transportation Systems, Vol. 7, No. 4, 429-436, doi:10.1109/tits.2006.884615

[18] Tientrakool, P.; Ho, Y.-C.; Maxemchuk, N. F. (2011). Highway capacity benefits from using vehicle-to-vehicle communication and sensors for collision avoidance, Proceedings of the 2011 IEEE Vehicular Technology Conference, 5 pages, doi:10.1109/vetecf.2011.6093130

[19] Lu, Q.; Tettamanti, T.; Hörcher, D.; Varga, I. (2019). The impact of autonomous vehicles on urban traffic network capacity: an experimental analysis by microscopic traffic simulation, Transportation Letters: The International Journal of Transportation Research, Vol. 12, No. 8, 540-549, doi:10.1080/19427867.2019.1662561

[20] Brown, A.; Gonder, J.; Repac, B. (2014). An analysis of possible energy impacts of automated vehicles, Meyer, G.; Beiker, S. (Eds.), Road Vehicle Automation, Springer, Cham, 137-153, doi:10.1007/978-3-319-05990-7_13

[21] Cao, H.; Zöldy, M. (2020). An investigation of autonomous vehicle roundabout situation, Periodica Polytechnica Transportation Engineering, Vol. 48, No. 3, 236-241, doi:10.3311/ PPtr.13762

[22] Taiebat, M.; Brown, A. L.; Safford, H. R.; Qu, S.; Xu, M. (2018). A review on energy, environmental, and sustainability implications of connected and automated vehicles, Environmental Science \& Technology, Vol. 52, No. 20, 11449-11465, doi:10.1021/acs.est.8b00127

[23] Barth, M.; Boriboonsomsin, K.; Wu, G. (2014). Vehicle automation and its potential impacts on energy and emissions, Meyer, G.; Beiker, S. (Eds.), Road Vehicle Automation, Springer, Cham, 103-112, doi:10.1007/978-3-319-05990-7 10

[24] Thomas, J.; Hwang, H.-L.; West, B.; Huff, S. (2013). Predicting light-duty vehicle fuel economy as a function of highway speed, SAE International Journal of Passenger Cars - Mechanical Systems, Vol. 6, No. 2, 859-875, doi:10.4271/2013-01-1113

[25] Carey, M.; Bowers, M. (2012). A review of properties of flow-density functions, Transport Reviews, Vol. 32, No. 1, 49-73, doi:10.1080/01441647.2011.608135

[26] Newell, G. F. (1993). A simplified theory of kinematic waves in highway traffic, part I: General theory, Transportation Research Part B: Methodological, Vol. 27, No. 4, 281-287, doi:10.1016/0191-2615(93)90038-C

[27] Gayah, V. V.; Gao, X.; Nagle, A. S. (2014). On the impacts of locally adaptive signal control on urban network stability and the macroscopic fundamental diagram, Transportation Research Part B: Methodological, Vol. 70, 255-268, doi:10.1016/j.trb.2014.09.010

[28] Cassidy, M. J.; Jang, K.; Daganzo, C. F. (2011). Macroscopic fundamental diagrams for freeway networks: Theory and observation, Transportation Research Record: Journal of the Transportation Research Board, Vol. 2260, No. 1, 8-15, doi:10.3141/2260-02

[29] Friedrich, B. (2016). The effect of autonomous vehicles on traffic, Maurer, M.; Gerdes, J. C.; Lenz, B.; Winner, H. (Eds.), Autonomous Driving, Springer, Berlin, 317-334, doi:10.1007/978-3-66248847-8_16

[30] Jones, S.; Philips, B. H. (2013). Cooperative adaptive cruise control: Critical human factors issues and research questions, Proceedings of the $7^{\text {th }}$ International Driving Symposium on Human Factors in Driver Assessment, Training, and Vehicle Design, 121-127, doi:10.17077/ drivingassessment.1477 